\title{
A Cross-Sectional Study \\ Investigating Patients' Preferences \\ Regarding Shared Decision Making in Adult Orthodontic Patients
}

F. Motamedi-Azari, F. S. Ryan, E. Jones and

S. J. Cunningham 


\begin{abstract}
Introduction

Shared decision making (SDM) is a process by which patients and clinicians work collaboratively to make decisions about healthcare.

Previously, research has shown that patients want to be more involved in decisions about their care but more recent evidence suggests that some patients may not wish to have the level of involvement which is now expected of them.
\end{abstract}

\title{
Aims
}

This study therefore investigated adult orthodontic patients' preferred and perceived roles in treatment decision making.

\section{Methods}

This cross-sectional study was carried out in a teaching hospital using the Control Preferences Scale (CPS). This involved asking patients to choose one of five cards relating, firstly, to their preferred role in orthodontic decision making and then their perceived role in their current orthodontic treatment decision making and these were compared.

\section{Results}

One-hundred patients were recruited and perceived roles in decision making tended to be more passive than patients said they preferred. Males were significantly more likely to select a passive role than females $(p=0.018)$.

\section{Conclusions}

Adult orthodontic patients perceived a more passive role in their current treatment decisions than they would have preferred. This highlights the importance of clinicians asking patients about their preferred role in treatment decision making from the outset. 


\section{Introduction}

The traditional paternalistic model of healthcare has been superseded in recent years, with patients being encouraged to take an active role in decisions about their own treatment. In England, this is evident in the often quoted mantra "no decision about me without me" which has been incorporated into the Health and Social Care Act and promotes the involvement of patients in decisions about their care. ${ }^{1}$ This concept is termed patient centred care (PCC), and shared decision making (SDM) is a key component of this.

Over the past decade, several key documents have outlined the importance of patient centredness and enhancing patient experiences. ${ }^{2,3,4}$ SDM has been defined as a collaborative process between clinicians and patients, where they work together to reach a mutual decision about a specific course of action or treatment. ${ }^{5,6 ;}$ it aims to empower patients and enables care to be tailored to the needs of the individual. ${ }^{5}$

$\mathrm{SDM}$ is now both an ethical and legal requirement, supported by professional regulatory bodies, including the General Medical and Dental Councils, and government organisations. ${ }^{6}$ There is evidence to suggest that the majority of patients want to be actively involved in their treatment and often want to be more involved than they currently are in making healthcare decisions. ${ }^{7-11}$ It has also been suggested that SDM may lead to better patient outcomes compared with situations where patients are passive recipients of care, and may result in fewer complaints and lower litigation rates. ${ }^{12}$ However, recent research by Fredriksson et al. ${ }^{13}$ presents conflicting evidence and suggests that not all patients want to be as involved to the same extent as clinicians think they do.

Stiggelbout and co-workers (2012) described the stages involved in SDM: an explanation of the nature of the problem, discussing the treatment options (including the option of undertaking no treatment), explaining the risks and benefits of each option and the use of decision aids. Next comes, exploring the patient's ideas, concerns and expectations regarding the treatment options and supporting the process of SDM; explaining that patients do not need to make the decision on their own. The clinician should then make recommendations based on the best available research evidence, along with their clinical experience and the patient's preferences and 
values. This facilitates SDM and the practice of evidence based dentistry. The patient's understanding should then be assessed and they should be given the opportunity to ask questions. SDM should not occur under time pressure and does not need to be in a single consultation, therefore for many patients arranging a review appointment gives them an opportunity to make a shared decision, by involving others and without time pressures. ${ }^{14}$

SDM can be measured in a number of different ways. Structured and objective instruments have been developed to enhance the measurement of SDM. A systematic review by Scholl et al (2011, cited by Da Silva, 2012) found eight instruments which measure patients' preferences for SDM and which have all undergone extensive psychometric testing. ${ }^{15,16}$ The most commonly used tool for measuring patients' preferences for involvement in SDM is the Control Preference Scale (CPS) (Degner and Beaton, 1987). ${ }^{17}$ This is a subjective measure of how much patients feel they have been involved in decisions about their healthcare and therefore measures one important component of SDM.

Research exploring SDM in dentistry is limited and it is therefore important to investigate how patients would like to be involved in treatment decision making and whether this matches with their perceived role in the decision making processes in their current care.

\section{$\underline{\text { Aims }}$}

The aims of this study were therefore to establish the following:

- Adult orthodontic patients' preferred role in decision making and also their perceived role in their decision making about their current orthodontic treatment

- To compare individual patient's preferred and perceived roles

\section{Patients and Methods}

This was a cross-sectional study carried out in a single teaching hospital (Eastman Dental Hospital, UCLH Foundation Trust) using a validated scale (the Control Preferences Scale). This involved face-to-face data collection from patients in orthodontic clinics. 
The study received ethical approval from the NHS Research Ethics Committee (Project ID: 18/NW/0592) on 23/08/2018. Written consent was obtained from all participants and the principles of the Declaration of Helsinki were followed.

Patients in this study included any adult patients (16 years and above) who were currently undergoing orthodontic treatment at the Eastman Dental Hospital, UCLH Foundation Trust and patients were recruited if they were in active fixed appliance treatment or in retention. Patients were recruited using convenience sampling and those with craniofacial syndromes or severe hypodontia with minimal orthodontic input were excluded, as it was thought that their perceptions may be different due to varying healthcare experiences. Patients with hypodontia involving standard orthodontic treatment and orthognathic patients were included in this study.

Although many orthodontic patients are adolescents, it was felt it would be more appropriate to investigate the concept of SDM with adult patients as they are making their own treatment decisions, unlike adolescents where parents often contribute to these decisions. The CPS was also developed for use with adult patients.

In preference to a formal sample size calculation, it was decided to recruit as many patients as possible during a 5-month period between October 2018 and February 2019, as there were a finite number of adult Orthodontic patients who met the inclusion criteria within the department.

The questionnaire utilised was the Control Preferences Scale (CPS) which was developed by Degner and Beaton (1987) $)^{17,18}$. As the CPS has been used in previous studies investigating SDM in dentistry ${ }^{19,20}$, it is therefore feasible to use and the data can be readily compared to other studies. The CPS measures patients' decision making preferences using 5 statements and each describes a different role in decision making: Most active, Active, Collaborative, Passive or Most passive (Table 1).

\begin{tabular}{|l|l|l|}
\hline Active Role & Collaborative Role & Passive Role \\
\hline $\begin{array}{l}\text { Most Active: "I prefer to } \\
\text { make the decision about } \\
\text { which treatment I will } \\
\text { receive" }\end{array}$ & $\begin{array}{l}\text { Collaborative: "I prefer } \\
\text { that my orthodontist and I } \\
\text { share responsibility for } \\
\text { deciding which treatment } \\
\text { is best for me" }\end{array}$ & $\begin{array}{l}\text { Passive: "I prefer that my } \\
\text { orthodontist makes the } \\
\text { final decision about which } \\
\text { treatment will be used, but } \\
\text { seriously considers my } \\
\text { opinion" }\end{array}$ \\
\hline
\end{tabular}




\begin{tabular}{|l|l|}
\hline $\begin{array}{l}\text { Active: "I prefer to make } \\
\text { the final decision about } \\
\text { my treatment after } \\
\text { seriously considering my } \\
\text { orthodontist's opinion" }\end{array}$ & $\begin{array}{l}\text { Most Passive: "I prefer to } \\
\text { leave all decisions } \\
\text { regarding treatment to my } \\
\text { orthodontist" }\end{array}$ \\
\hline
\end{tabular}

Table 1 - The 5 statements that form the Control Preferences Scale for patients' preferred roles

Potential participants were approached by their own clinician, who was not a member of the research team, at one of their routine visits and the study was briefly explained to them. If they wished to consider being involved they were then introduced to the researcher to explain the study in more detail. As detailed in the results section, 1 out of 101 participants declined involvement in the study.

Patients were initially asked to choose the card that best described their preference regarding decision making in their own orthodontic care (Figure 1) and they were then asked to choose the card that best described their perceived role in their current orthodontic treatment (Figure 2). This allowed for their preferred and current perceived roles to be compared. Patients completed the CPS during their active orthodontic treatment or retention phase.

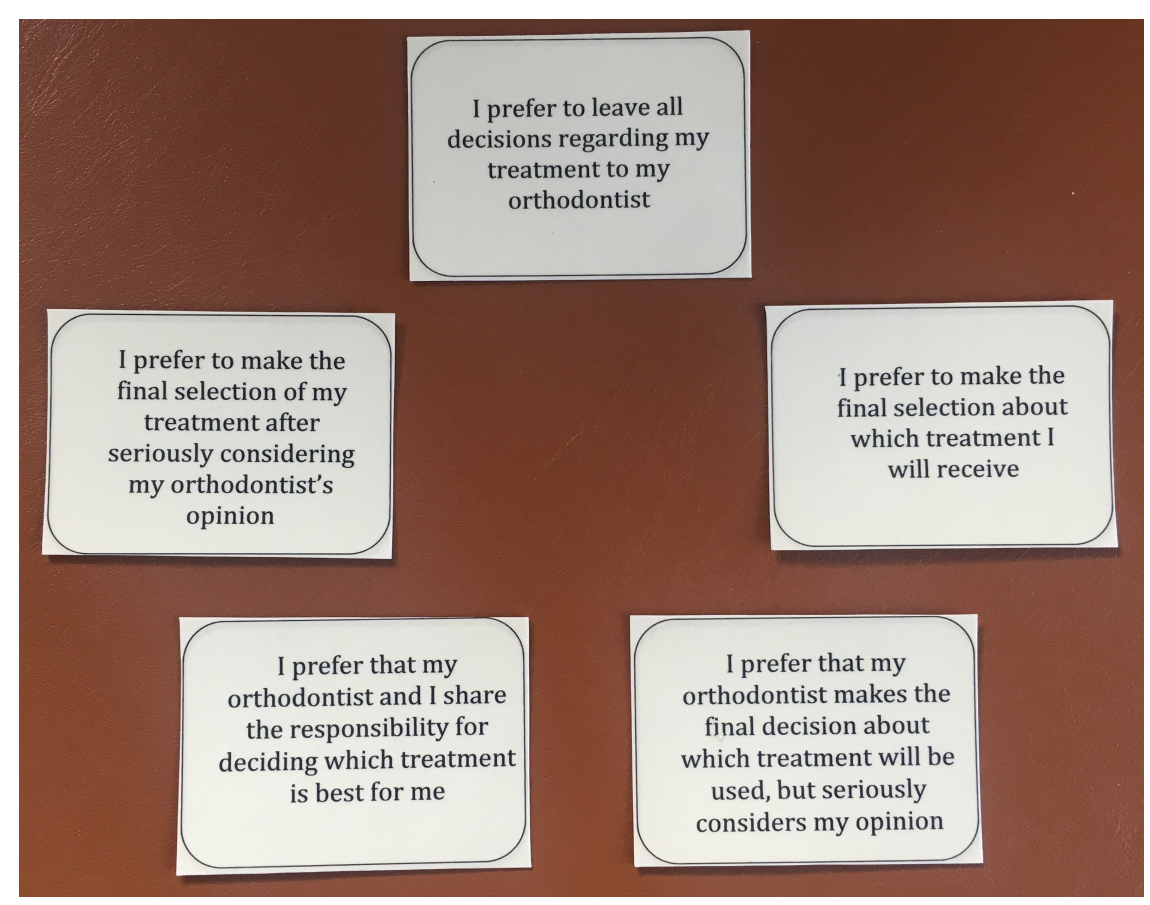

Figure 1 - Control Preferences Scale (CPS) cards related to preferred role 


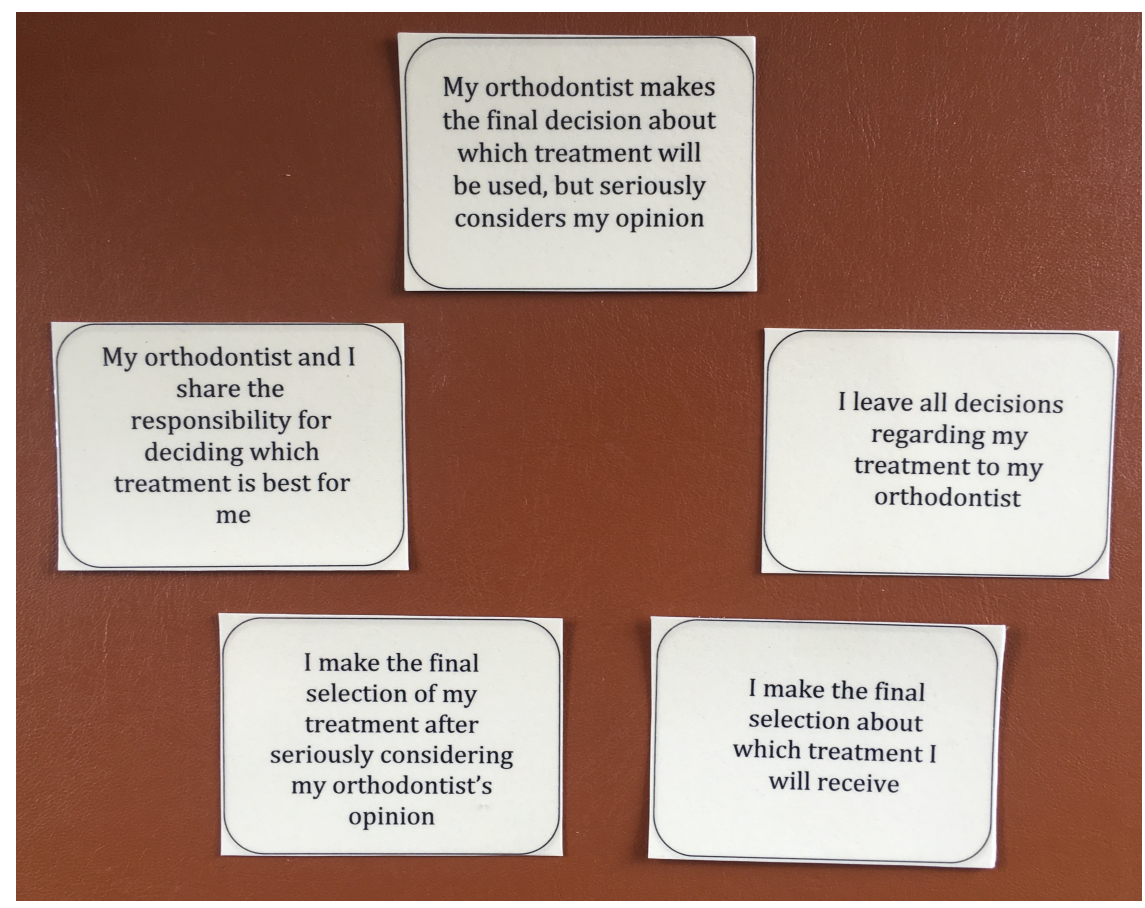

\section{Figure 2 - Control Preferences Scale (CPS) cards related to perceived role}

The data collected were recorded manually into a data collection table by a single researcher and then transferred to the SPSS statistical package version 22 for analysis. Other information recorded included age, gender and stage of treatment (whether the patient was in active treatment or in retention).

The demographic information and patients' preferred and perceived roles were analysed using descriptive statistics. The data were also analysed to assess if age, gender or stage of treatment had an effect on patients' role preferences. For categorical data (gender and stage of treatment), the non-parametric Fisher's exact test was used and the continuous data (age) were analysed using the Kruskal Wallis test. The agreement between patients' preferred and perceived roles in treatment decision making was analysed using weighted kappa on the $\mathrm{R}$ statistical software, as these role categories were ordered. For example, an Active preferred role and a Collaborative perceived role is "better" than an Active preferred role and a Passive perceived role.

\section{Results}

In total, 101 patients were approached; 1 patient declined involvement but the remaining 100 were recruited into the study. There was a higher percentage of female 
patients (58\%) compared with males (42\%). The mean age of the patients was 22.53 years old (SD 10.03 years), with a large age range between 16 and 73 years of age. The study sample included patients with all incisor relationships, with Class II division 1 (30\%) being the most frequent, closely followed by Class I (29\%), Class III $(22 \%)$ and Class II division 2 (19\%). The majority of the patients were in active treatment (76\%), with $24 \%$ having completed treatment and currently in retention.

Preferred role: The results showed that the most commonly selected options for preferred role were collaborative $(31 \%)$, and passive $(29 \%)$. A similar number selected an active role $(20 \%)$ or most passive (19\%) role. Only $1 \%$ said they preferred the most active role. Due to the fact that there was only 1 person in the most active group, this group was combined with the active group for statistical analysis.

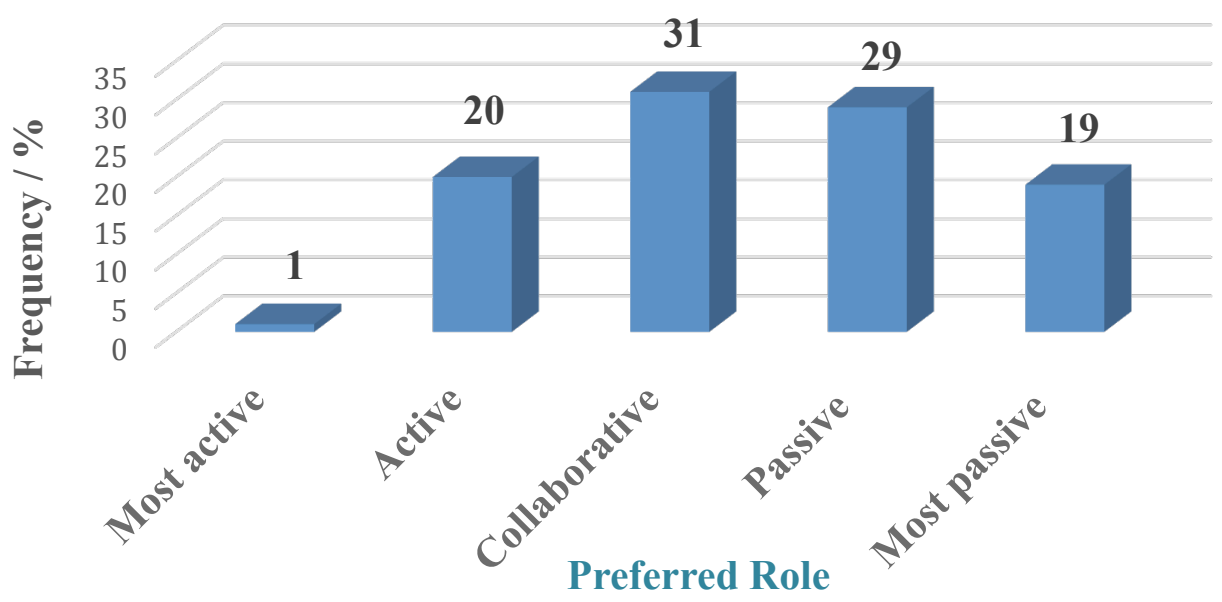

\section{Figure 3 - Bar chart illustrating patients' preferred roles in treatment decision making.}

Perceived role: The results showed that, $36 \%$ of patients perceived their role in their current orthodontic treatment to be passive or most passive $(32 \%)$. Only $20 \%$ of patients perceived that the approach to decision making in their current treatment was collaborative and $12 \%$ felt they had an active role. No patients perceived their role to be most active. 


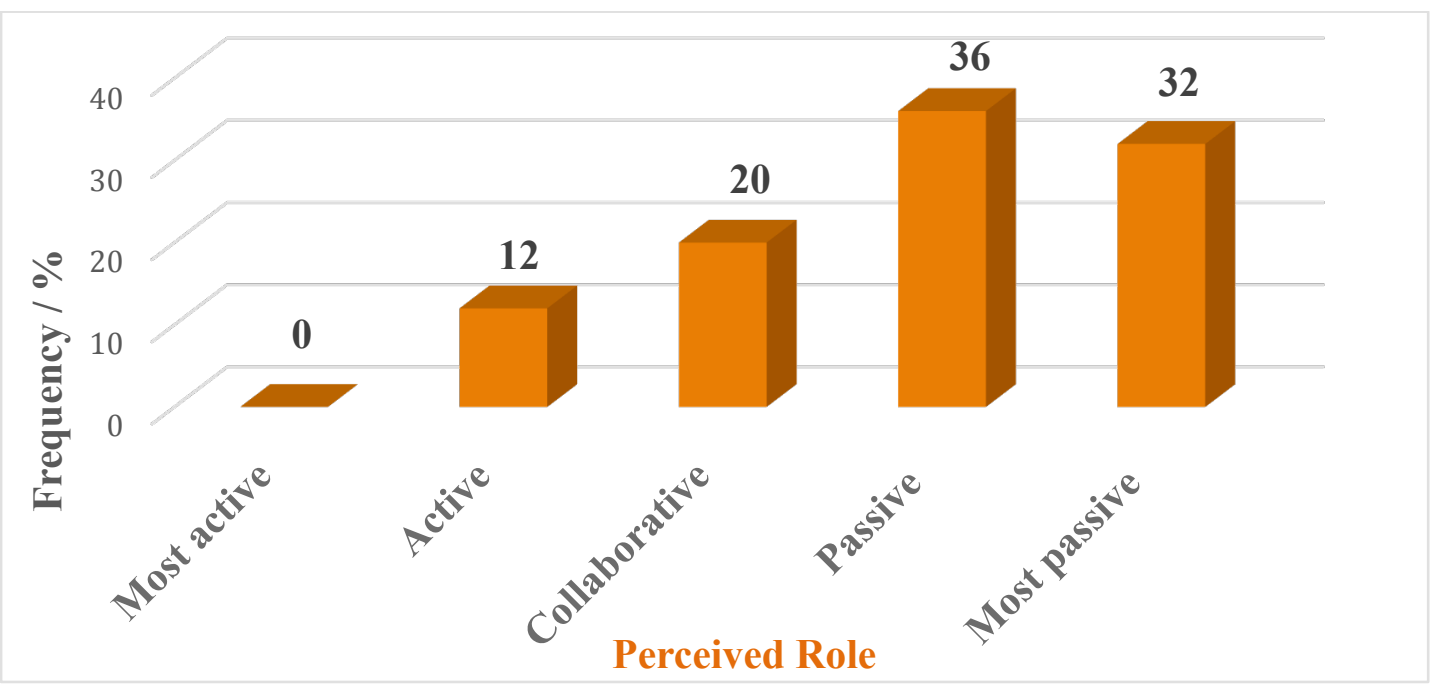

Figure 4 - Bar chart illustrating patients' perceived roles in treatment decision making.

There was only fair agreement $(\mathrm{k}=0.32)$ between patients' preferred and perceived roles in treatment decision making.

The data were then analysed for each individual patient and this showed that, for $45 \%$ of patients, their preferred and perceived roles did match, however for $55 \%$ they did not. Of the 55 patients whose roles did not match, 39 (71\%) wanted a more active role and $16(29 \%)$ wanted a more passive role in decision making about their orthodontic treatment.

Factors which may potentially affect choice of role preference were then investigated:

Stage of Treatment: In the active treatment group, the most commonly selected preferred role was collaborative (31.6\% [24/76]). In contrast, the most commonly selected role in the retention group was passive (41.7\% [10/24]). There was, however, no statistically significant difference between patients' preferred roles and their stage of treatment $(\mathrm{p}=0.439)$.

Gender: There was a statistically significant difference $(\mathrm{p}=0.018)$ between males and females for preferred role in decision making (Table 2). For female patients, the most commonly selected option was a collaborative role at $43 \%(25 / 58)$. In contrast, the option selected most frequently by males was passive at 38\% (16/42). 


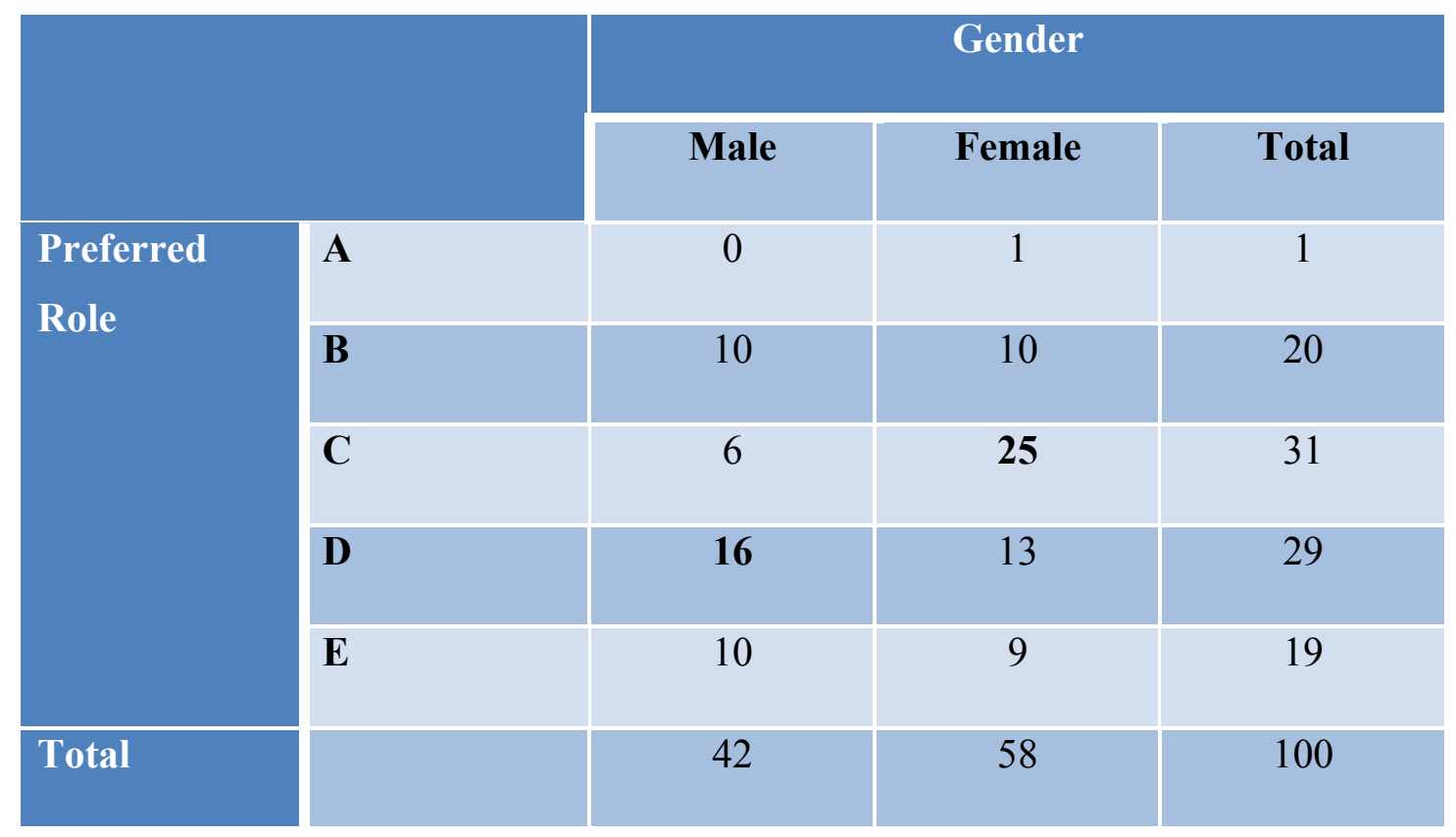

Table 2 - Patients' preferred role according to gender

Age: There was no evidence of differences in age among the 5 preferred role categories $(p=0.680)$, however the small number of older patients must be highlighted.

\section{Discussion}

This study investigated SDM in adult orthodontic patients; the sample size $(n=100)$ was good and representative of those adult patients undergoing orthodontic treatment at the Eastman Dental Hospital. This is because the findings for gender (female: male ratio) and the distribution of malocclusions treated are in keeping with this population group. However, as this is a single centred study it is limited in-terms of its generalisability. The fact that a significant difference was found when comparing preferred role and gender suggests that an adequate sample size was recruited for this element of the study. It may be beneficial to have higher numbers for the other subgroup analyses in future work of this type however.

The literature has shown that many factors may affect SDM including age, gender, education level, socio-economic status (SES), ethnicity, country of birth, marital and employment status. ${ }^{8}$ Patients in the current study were asked their age and gender, but 
other data were not collected. These would however be interesting areas for further research.

The results showed that $31 \%$ of patients said they preferred a collaborative role in treatment decision making, closely followed by a passive role $(29 \%)$. This is similar to the results of Chapple et al. ${ }^{19}$ where they found that $40 \%$ of patients preferred a collaborative role in decision making for general dental treatment in UK hospital and general dental practice settings. The findings are also similar to the results of a recent study by Reissmann et al. ${ }^{20}$ in Germany, who found that $51.5 \%$ of patients preferred a collaborative role in decision making for general dental treatment, followed by $27.3 \%$ who preferred a passive role. These findings are interesting as they indicate that not all patients want a truly collaborative role in their healthcare as many authorities have previously believed. Other patients may prefer a passive role in their care, where the clinician plays a greater role in decision making, whilst also considering the patient's wishes or indeed prefer an active role where they take a greater role in decision making, whilst considering the clinician's input. This variation highlights the importance of discussing the patient's preferred approach at the start of treatment so that the clinician is able to support them appropriately. There is also benefit in explaining to patients why a collaborative approach may be beneficial, highlighting improved outcomes and enhanced satisfaction in those who engage in truly shared decision making.

The results of this current study showed that the least preferred options were most passive $(19 \%)$ and most active (1\%) roles. Again, this is similar to the findings of Chapple et al. ${ }^{19}$ and Reissmann et al. ${ }^{20}$ who noted that the extreme role preferences were the least preferred options. This may be because patients prefer not to have complete control, or indeed no control, in decision making about their own care and recognise the importance of having some input from both patient and clinician.

Of the patients in this study, only $20 \%$ perceived their actual role in their current treatment to be collaborative, $36 \%$ felt their role was passive and $32 \%$ mostly passive, with only $12 \%$ selecting active and none considering their role to be most active. Again, this was similar to the findings in the Chapple et al. ${ }^{19}$ study where the majority of patients perceived their role to be passive (40\%) or most passive (28\%). Reissmann 
et al. $^{20}$ reported that the majority of patients perceived their role to be passive (37.4\%), followed by collaborative $(29.3 \%)$.

There was only fair agreement between patients' preferred and perceived roles in decision making $(\mathrm{k}=0.32)$. For $45 \%$ of patients in this study, their preferred role matched their perceived role and, interestingly this finding was almost identical to that of Reissmann et al. ${ }^{20}$ who reported that preferred and perceived roles matched in $45.5 \%$ of their patients.

This suggests that some patients may still be treated under a paternalistic model of care and some clinicians may not be engaging in SDM, or may think that they are involving patients in treatment decisions more than they actually are. This is important because a paternalistic model of healthcare is no longer supported by any professional bodies or government organisations, as it is considered unethical. ${ }^{6}$ Alternatively, patients may not be picking up cues which would allow them to be involved and clinicians may need to be clearer in their communication.

However, for $55 \%$ of patients in the current study, patients' preferred and perceived roles did not match; of those 55 patients, 39 (71\%) said they would prefer a more active role, compared with 16 (29\%) who said they would prefer a more passive role. The findings of this study were again similar to the Reissmann et al. ${ }^{20}$ study who found that of the $54.5 \%$ of patients whose preferred and perceived roles did not match, the majority (91\% [49.5/54.5]) wanted a more active role.

Interestingly, $16 \%$ of patients in this study sample said they would prefer a more passive role in decision making compared with their perceived current role, which highlights that not all patients want to be as actively involved as clinicians may think they do and, again, this highlights the importance of discussing these aspects of care with patients. A number of these patients felt that they did not have the knowledge of Orthodontics to be making these decisions, therefore clinicians may need to encourage these patients to be involved to help them make informed decisions. The evidence suggests that this may be associated with better outcomes and higher levels of patient satisfaction. ${ }^{16,21}$ Clinicians may do this by explaining to patients with passive role preferences the benefits of taking a more collaborative or active role in decision making and asking about their reasons for choosing this role preference. This means that clinicians may need to be more mindful of patients who prefer a passive 
role and spend more time encouraging them and explaining the benefits of shared decision making.

When the effects of stage of treatment, gender and age were analysed, stage of treatment did not appear to significantly affect patients' preferred role in orthodontic decision making. This may be because once patients have started treatment their preferences are already established and do not tend to change during treatment. However, as the number of patients in the retention group was small $(n=24)$, this may also have precluded any differences being identified and it would be interesting to investigate this further in future studies, with a larger number of patients in this group. Although patients were asked about their experience to date, they are more likely to remember more recent experiences, which may have introduced some bias in the results. It would be interesting to investigate patients' preferred and perceived roles across different aspects of treatment in future studies.

The literature suggests that there are gender differences for patients' role preferences, with female patients generally preferring more active, and males more passive roles. ${ }^{22}$ The results of the current study supported this hypothesis, with males tending to prefer more passive roles (Table 2) and this gender difference was statistically significant $(\mathrm{p}=0.018)$. These findings were also supported by Hanna et al. ${ }^{22}$ who found that female patients were significantly $(\mathrm{p}=0.04)$ more likely to prefer an active role with regards to decision making for third molar extractions. This suggests that clinicians should be mindful of the relationship between gender and patients' role preferences when discussing options. It may be, for instance, that females require more information or more time to consider the treatment options than males.

The literature also suggests that there is a relationship between patient age and their preferred roles, with younger patients preferring more active roles. ${ }^{23}$ However, the results of this study did not show a statistically significant difference between age and preferred role $(\mathrm{p}=0.680)$. This may be because, although a wide age range of patients were recruited (16-73 years old), the majority were at the lower end of this range (mean age 22.53 years old), therefore as the study sample generally represented younger patients, any potential associations may not be detected. It would therefore be interesting to investigate this in future studies with more patients in the older age group. 
The attitudes and roles of healthcare professionals towards patient involvement may also influence their approach to SDM. One of the benefits of recruiting patients from a large number of clinicians, as was the case in this study, was that the results captured different clinicians' personalities and therefore enhanced the generalisability. Although the CPS has its limitations in-terms of how much can be captured from simple statements, it however allows for some quantification of how well patients feel they have been involved. In hindsight all multi-disciplinary patients should have been excluded as they may have had previous exposure to healthcare, which may have introduced some bias in the results. The grade of the clinicians involved in the decision making process was not recorded in this study, however this would be an interesting area for future research to assess if this has an affect on patients' perceived roles.

The findings of this study highlight the importance of clinicians asking patients about their preferred role in treatment decision making from the outset and not assuming that everyone wants the same level of involvement. Furthermore, encouraging some patients to have a more active role may be important in enhancing treatment outcomes and satisfaction. This study also emphasises the importance of training and education in SDM amongst clinicians and patients, in order to standardise and improve the delivery and quality of care our patients' receive.

\section{Conclusions}

- The most common preferred choices in orthodontic decision making were collaborative (31\%) and passive (29\%), but the most common perceived choices were passive (36\%) and most passive (32\%).

- There was only fair agreement between adult patients' preferred and perceived roles in orthodontic decision making overall $(\mathrm{k}=0.32)$.

- A significant gender difference $(p=0.018)$ was found in this study, with males preferring more passive roles than females in treatment decision making.

3 In brief points: 
- Findings highlight the importance of clinicians asking patients about their preferred role in treatment decision making from the outset

- Encouraging some patients to have a more active role may be important in enhancing treatment outcomes and satisfaction

- Further training and education in SDM amongst clinicians and patients is required to improve the overall quality of patient care

\section{Declaration of Interests}

None to declare.

\section{Acknowledgements}

We would like to thank all of the patients who were involved in this study.

\section{References}

1. Department of Health. Health and Social Care Act. Great Britain: The Stationery Office, 2012.

2. Darzi A. Quality and the NHS next stage review. The Lancet 2008; 371(9624):1563-1564.

3. Department of Health. The NHS Constitution. London: Department of Health, 2009.

4. Department of Health. Equity and excellence: liberating the NHS. London: The Stationery Office, 2010.

5. The Health Foundation. Person-centred care made simple. What everyone should know about person-centred care. 2014. Online information available at: http://personcentredcare.health.org.uk/sites/default/files/resources/personcentred_care_made_simple_1.pdf(Accessed March 2018).

6. Coulter A, Collins A. Making shared decision-making a reality. London: King's Fund. 2011. 
7. Scheibler F, Janssen C, Pfaff H. Shared decision making: an overview of international research literature. Sozial-und Praventivmedizin. 2003;48(1):1123.

8. De Silva D. Helping people share decision making. The Health Foundation; 2012

9. Thompson AG. The meaning of patient involvement and participation in health care consultations: a taxonomy. Social Science \& Medicine. 2007;64(6):1297-310.

10. Chewning B, Bylund CL, Shah B, Arora NK, Gueguen JA, Makoul G. Patient preferences for shared decisions: a systematic review. Patient Education and Counselling. 2012;86(1):9-18.

11. Couët N, Desroches S, Robitaille H, et al. Assessments of the extent to which health-care providers involve patients in decision making: a systematic review of studies using the OPTION instrument. Health Expectations. 2015;18(4):542-61.

12. Légaré F, Stacey D, Turcotte S, et al. Interventions for improving the adoption of shared decision making by healthcare professionals. Cochrane Database of Systematic Reviews. 2014.

13. Fredriksson M, Eriksson M, Tritter J. Who wants to be involved in health care decisions? Comparing preferences for individual and collective involvement in England and Sweden. BMC Public Health. 2018;18(1):18.

14. Stiggelbout AM, Van der Weijden T, De Wit MP, et al. Shared decision making: really putting patients at the centre of healthcare. British Medical Journal. 2012;344:256.

15. Scholl I, Koelewijn-van Loon M, Sepucha K, et. al. Measurement of shared decision making-a review of instruments. Zeitschrift für Evidenz, Fortbildung und Qualität im Gesundheitswesen. 2011;105(4):313-24.

16. Da Silva D. Helping people share decision making: a review of evidence considering whether shared decision making is worthwhile. The Health Foundation; 2012.

17. Degner LF, Beaton JI. Life-death decisions in health care. 1987.

18. Degner LF, Sloan JA, Venkatesh P. The control preferences scale. Canadian Journal of Nursing Research Archive. 1997;29(3). 
19. Chapple H, Shah S, Caress AL, Kay EJ. Exploring dental patients' preferred roles in treatment decision-making-a novel approach. British Dental Journal. 2003;194(6):321

20. Reissmann DR, Bellows JC, Kasper J. Patient Preferred and Perceived Control in Dental Care Decision Making. JDR Clinical \& Translational Research. 2019;4(2):151-9.

21. Légaré F, Stacey D, Turcotte S, et al. Interventions for improving the adoption of shared decision making by healthcare professionals. Cochrane database of systematic reviews. 2014(9).

22. Hanna K, Sambrook P, Armfield JM, Brennan DS. Preferences for dental decisional control and associations with quality of life among third molar patients attending public dental services. Community Dental Health. 2017;34(3):163-8.

23. Tricou C, Yennu S, Ruer M, Bruera E, Filbet M. Decisional control preferences of patients with advanced cancer receiving palliative care. Palliative \& Supportive Care. 2018;16(5):544-51. 